HD-THEP-03-60

\title{
Towards a renormalizable standard model without fundamental Higgs scalar
}

\author{
Holger Gies ${ }^{1}$, Joerg Jaeckel ${ }^{2}$ and Christof Wetterich ${ }^{3}$ \\ Institut für theoretische Physik, Universität Heidelberg, \\ Philosophenweg 16, D-69120 Heidelberg, Germany \\ 1 E-mail: h.gies@thphys.uni-heidelberg.de \\ 2 E-mail: j.jaeckel@thphys.uni-heidelberg.de \\ 3 E-mail: c.wetterich@thphys.uni-heidelberg.de
}

\begin{abstract}
We investigate the possibility of constructing a renormalizable standard model with purely fermionic matter content. The Higgs scalar is replaced by point-like fermionic self-interactions with couplings growing large at the Fermi scale. An analysis of the UV behavior in the point-like approximation reveals a variety of non-Gaußian fixed points for the fermion couplings. If real, such fixed points would imply nonperturbative renormalizability and evade triviality of the Higgs sector. For point-like fermionic self-interactions and weak gauge couplings, one encounters a hierarchy problem similar to the one for a fundamental Higgs scalar.
\end{abstract}

\section{Introduction}

The standard model of elementary particle physics is remarkably successful in describing physics up to a scale of the order of several hundred GeV. Still it faces a number of shortcomings, such as the abundance of parameters and their origin, which become particularly prominent in flavor physics or neutrino physics. In addition, there are two problems that appear to point more strongly to the fact that the standard model cannot be a fundamental theory valid for arbitrarily short distance scales: first, the problem of triviality of the scalar and abelian gauge sector, and second, the gauge hierarchy problem.

Let us start with the hierarchy problem. If one assumes that the standard model is valid up to some high scale $\Lambda_{\mathrm{UV}}$ (e.g., a scale of grand unification, $\mathrm{M}_{\mathrm{GUT}} \sim 10^{16} \mathrm{GeV}$, or even the Planck scale), one is immediately confronted with two immensely different scales in the theory - the electroweak symmetry-breaking scale $\mathrm{M}_{\mathrm{EW}} \sim 100 \mathrm{GeV}$ and $\Lambda_{\mathrm{UV}}$. A realization of this enormous hierarchy in the context of the standard model requires a highly exceptional choice of parameters ("fine-tuning"). This can be seen from the quadratic renormalization of the scalar mass term in the Higgs sector which naively receives corrections of the or$\operatorname{der} \Lambda_{\mathrm{UV}}^{2} \gg m_{\mathrm{Higgs}}^{2}$ when quantum fluctuations between $\Lambda_{\mathrm{UV}}$ and $\mathrm{M}_{\mathrm{EW}}$ are integrated out. Thus $m_{\text {Higgs }}$ at the UV scale must be extremely fine-tuned in order to cancel most of the quantum corrections and produce a Higgs which is much lighter than $\Lambda_{\mathrm{UV}}$. It should be stressed that the hierarchy problem is not a problem of principle, but rather a problem of 
likeliness. Furthermore, it is sufficient that a suitable renormalized parameter at the scale $\Lambda_{\mathrm{UV}}$ be close to a particular critical value, i.e., (partial) fixed point. No fine-tuning "order by order" is necessary in a suitable renormalization group treament [1]. Also the existence of the (partial) fixed point is related to an enhanced (approximate) dilatation symmetry of the quantum system and therefore natural. Nevertheless, the necessity of an explanation for a "close-to-critical value" at $\Lambda_{\mathrm{UV}}$ remains.

Triviality of the scalar and the abelian sector is a fundamental problem. If we require a theory to be valid up to arbitrarily high momentum scales, we have to take the limit $\Lambda_{\mathrm{UV}} \rightarrow \infty$. Trivial quantum field theories become noninteracting in this limit, since their renormalized couplings vanish. Viewed differently, if we start, for instance, with an interacting Higgs sector at low energies, the (perturbative) running of scalar coupling approaches a singularity (Landau pole), signaling the possible break-down of the theory and the onset of new physics. Triviality in the flow of a renormalized coupling that is observed experimentally at a nonzero value experimentally unequivocally means that the standard model cannot be continued to arbitrarily short distances. This obvious shortcoming of a "fundamental standard model" is often not considered as a major problem in practice: unification with gravity suggests that the standard model has to be extended at the Planck scale anyhow.

In many attempts at formulating theories beyond the standard model, the hierarchy and triviality problem are not considered simultaneously. For the hierarchy problem, new physical mechanisms are invoked to already operate on a low scale, say $\mathcal{O}(\mathrm{TeV})$, in order to strongly modify the renormalization group (RG) behavior of the system. Prominent examples of these scenarios are supersymmetry 2, technicolor [3] or the "little Higgs" 44. Common to all of these is the need of additional physical parameters instead of their desired reduction. As the main hope, no parameter needs to be fine-tuned at the high scale $\Lambda_{\mathrm{UV}}$ and the system is (marginally) RG stable towards the infrared (IR). The triviality problem is finally circumvented by embedding the standard model in a nontrivial theory (as the abelian $\mathrm{U}(1)$ is embedded in a nonabelian gauge group of a GUT), or by referring to a framework that goes far beyond quantum field theory, such as string theory.

This letter represents an attempt at tackling these problems from a different viewpoint. Instead of modeling new physics with additional parameters at the TeV scale (IR viewpoint), we are looking for quantum field theoretic systems defined at a high scale $\Lambda_{\mathrm{UV}}$ that have the potential to remain RG stable down to low scales (UV viewpoint). The desired systems should not have more relevant parameters than the standard model; in particular, no new parameters should be required for the system to become unstable near the Fermi scale (as is the case, e.g., with soft-breaking terms in SUSY theories).

The hierarchy problem is deeply rooted in the scalar nature of the Higgs sector. In the present work we avoid the concept of fundamental scalars and consider only fermionic matter fields. The Higgs scalar is then interpreted as a fermionic bound state, whereas the Higgs condensate arises from dynamical symmetry breaking accompanied by a condensation of fermion bilinears. This behavior is well known from the Nambu-Jona-Lasinio (NJL) model [5] and systems alike, which have already frequently been used for modeling the Higgs sector, as in topquark-condensation scenarios [6]. In fact, from a model-building 
point of view, our systems closely resemble those with topquark condensation. However, we focus on the UV behavior, which is an inherently nonperturbative problem.

At first sight, it seems that fermionic self-interactions even worsen the theoretical objections, since such couplings are not perturbatively renormalizable in $D=4$ dimensional spacetime. This means that a quantum theory cannot consistently be constructed by an expansion around zero coupling (Gaußian fixed point). Nevertheless, perturbative renormalizability is not a necessary criterion for constructing an interacting field theory. Also perturbatively nonrenormalizable theories can be fundamental and mathematically consistent down to arbitrarily small length scales, as proposed in Weinberg's "asymptotic safety" scenario [7]. This scenario assumes the existence of a non-Gaußian (=nonzero) UV fixed point under the renormalization group ( $R G$ ) operation at which the continuum limit can be taken. The theory is "nonperturbatively renormalizable" in Wilson's sense. If the non-Gaußian fixed point is IR repulsive only for a finite number of renormalized couplings, the RG trajectories along which the theory can flow for $M_{\mathrm{EW}} / \Lambda_{\mathrm{UV}} \rightarrow 0$ are labeled by only a finite number of physical parameters. Then the theory is as predictive as any perturbatively renormalizable theory, and high-energy physics can be well separated from low-energy physics without tuning a large number of parameters. Finally, the triviality problem is absent by construction.

The issue of the gauge hierarchy problem is related to the relevant couplings and the associated anomalous dimensions. For definiteness, let us consider one particular small deviation $^{1} v$ from the fixed point which depends on the renormalization scale $k$ according to a generalized "anomalous dimensions" $\Theta$,

$$
\partial_{t} v=k \partial_{k} v=-\Theta v
$$

The solution $v \sim k^{-\Theta}$ implies for large positive $\Theta$ that $v$ must be tiny at $\Lambda_{\mathrm{UV}}$ if it is of order one for $k=M_{\mathrm{EW}}$. This is the fine-tuning problem. For positive $\Theta$ (not very close to zero) $v$ is called relevant parameter and we conclude that a fine-tuning problem is connected to every relevant parameter. (We note that for a perturbative expansion the exact location of the fixed point may depend on the order of the approximation. Also $\Theta$ may be expressed in a perturbative series and the right-hand side may contain higher powers of $v$. All this does not affect the conclusion that precisely one small parameter $v\left(\Lambda_{\mathrm{UV}}\right)$ is needed (one fine-tuning) for every relevant coupling.) On the other hand, if $\Theta$ is very close to zero or vanishes (in this case $v$ may depend logarithmically on $k$ ), $v$ is called a marginal coupling. (An example is the gauge coupling $g$ near a fixed point with $g=0$.) No extreme fine-tuning is needed for marginal couplings, since $v\left(M_{\mathrm{EW}}\right)$ and $v\left(\Lambda_{\mathrm{UV}}\right)$ are of a similar order of magnitude. In consequence, a model with only marginal couplings has no gauge hierarchy problem. Within the standard model, this type of solution for the gauge hierarchy problem has been proposed in [8]. Known examples for the quantum field theories with only marginal couplings (besides the irrelevant ones) are four-dimensional pure non-abelian gauge theories near the Gaußian fixed point or two-dimensional scalar

\footnotetext{
${ }^{1}$ More precisely, $v$ is an eigenvector of the stability matrix. We take $v$ to be a dimensionless renormalized parameter. In the case of a mass $M$, this corresponds to $M / k$.
} 
theories with global U(1) symmetry near the fixed point corresponding to the KosterlitzThouless phase transition with essential scaling [9, 10. In statistical physics, a fixed point with only marginal directions can be associated to "self-organized criticality".

In this work, we analyze a class of models with four-fermion self-interactions with gauge and flavor symmetry. In order to quantize the systems in a nonperturbative framework, we employ the exact renormalization group formulated in terms of a flow equation for the effective average action $\Gamma_{k}[1]$,

$$
\partial_{t} \Gamma_{k}=\frac{1}{2} \operatorname{STr} \partial_{t} R_{k}\left(\Gamma_{k}^{(2)}+R_{k}\right)^{-1}, \quad t=\ln \frac{k}{\Lambda_{\mathrm{UV}}} .
$$

The latter is a free-energy functional that interpolates between the bare action $\Gamma_{k=\Lambda_{\mathrm{UV}}}=S$ and the full quantum effective action $\Gamma_{k=0}$. Here, $R_{k}$ denotes a to some extent arbitrary regulator function that specifies the details of the momentum-shell integrations. With the flow equation (2), it is possible to analyze the space of action functionals and its fixed-point structure, in order to look for quantizable and renormalizable theories. These correspond to zeros of the right-hand side of Eq. (2) with a suitable finite number of relevant parameters, characterizing the small deviations from the fixed point. Equation (2) is a functional differential equation, since the right-hand side contains the second functional derivative $\Gamma_{k}^{(2)}$ (the full inverse propagator). Approximate solutions can be found by suitable truncations of the space of actions.

Within the approximation of point-like four-fermion interactions, we indeed find a variety of non-Gaußian fixed points that give rise to new universality classes of interacting quantum field theories and solve the triviality problem in the Higgs sector. Concerning the hierarchy problem, however, we show that these fermionic theories are at least as IR unstable as a scalar Higgs sector, so that a very precise choice of initial conditions remains necessary (fine-tuning).

Upon the inclusion of gauge field dynamics, the picture does not change qualitatively as long as the gauge couplings remain perturbatively small. The influence of the gauge fields on the fermionic sector is subdominant. Moreover, the fermionic self-interactions do not modify the leading-order running of the gauge couplings, as we show with the aid of modified Ward-Takahashi identities. As a consequence, fermionic self-interactions cannot cure the triviality problem of the abelian $\mathrm{U}(1)$ sector, e.g., by rendering this gauge coupling asymptotically free.

While we have not found a solution to the gauge hierarchy problem so far, we reveal instructive general aspects of the structure and influence of fermionic self-interactions in models with flavor and gauge symmetry. Our findings may be taken as a hint toward possible directions in the search for a satisfactory renormalizable standard model without a fundamental Higgs scalar. As a rather speculative example, we demonstrate in the appendix how the possible existence of a non-Gaußian fixed point in the U(1) gauge coupling could strongly influence and even stabilize the RG behavior of a fermionic Higgs sector towards the infrared. 


\section{Toy Modeling the Standard Model}

In the present work, we consider a theory of fermions with self-interactions as well as gauge-field interactions. Let us start with all possible interactions that are compatible with a $\mathrm{U}(1) \times \mathrm{SU}\left(\mathrm{N}_{\mathrm{c}}\right)$ gauge symmetry and a chiral $\mathrm{SU}\left(\mathrm{N}_{\mathrm{f}}\right)_{\mathrm{L}} \times \mathrm{SU}\left(\mathrm{N}_{\mathrm{f}}\right)_{\mathrm{R}}$ flavor symmetry for $\mathrm{N}_{\mathrm{f}}$ fermion species. The $\mathrm{SU}\left(\mathrm{N}_{\mathrm{c}}\right)$ simulates the non-abelian, asymptotically free part of the standard model gauge group, while the $\mathrm{U}(1)$ models the abelian part with its triviality problem. For simplicity, the gauge fields are assumed to couple to left- and right-handed fermions in the same way with the same charges. In comparison to the standard model, we have neglected the electroweak $\mathrm{SU}(2)_{\mathrm{L}}$ chiral gauge interactions and the differences between the hyper charges under the $\mathrm{U}(1)$. The reason for concentrating on the strong $\mathrm{SU}\left(\mathrm{N}_{\mathrm{c}}\right)$ gauge group instead of the electroweak $\mathrm{SU}(2)_{\mathrm{L}}$ is the following: if an IR stabilized system exists, we expect the strongest gauge interaction to be a good candidate for a destabilizing influence near the Fermi scale. Even though symmetry breaking first affects the electroweak sector, it may be caused by fermionic self-interactions in combination with the strong gauge sector. An inclusion of the remaining standard model building blocks is, in principle, straightforward: one should add the weak gauge interactions and consider additional possible four-fermion interactions which are consistent with the reduced flavor symmetries and the gauge symmetries. The number of possible four-fermion interactions increases very substantially, however, owing to the lack of parity symmetry and reduced flavor symmetry. For $\mathrm{N}_{\mathrm{c}}=3, \mathrm{~N}_{\mathrm{f}}=6, \bar{e}=0$ our model corresponds to the standard model in the limit of vanishing weak and hypercharge gauge couplings.

We concentrate on a simple truncation with point-like couplings and include all possible four-fermion interactions obeying an $\mathrm{SU}\left(\mathrm{N}_{\mathrm{c}}\right) \times \mathrm{U}(1)$ gauge symmetry and an $\mathrm{SU}\left(\mathrm{N}_{\mathrm{f}}\right)_{\mathrm{L}} \times$ $\mathrm{SU}\left(\mathrm{N}_{\mathrm{f}}\right)_{\mathrm{R}}$ flavor symmetry ${ }^{2}$,

$$
\begin{aligned}
\Gamma_{k}= & \int \bar{\psi}\left(\mathrm{i} Z_{\psi} \not \partial+Z_{1} \bar{g} A+Z_{1}^{\mathrm{B}} \bar{e} \not B\right) \psi+\frac{Z_{\mathrm{F}}}{4} F_{z}^{\mu \nu} F_{\mu \nu}^{z}+\frac{Z_{\mathrm{B}}}{4} B^{\mu \nu} B_{\mu \nu}+\frac{\left(\partial_{\mu} A^{\mu}\right)^{2}}{2 \alpha}+\frac{\left(\partial_{\mu} B^{\mu}\right)^{2}}{2 \alpha_{B}} \\
& +\frac{1}{2}\left[Z_{-} \bar{\lambda}_{-}(\mathrm{V}-\mathrm{A})+Z_{+} \bar{\lambda}_{+}(\mathrm{V}+\mathrm{A})+Z_{\sigma} \bar{\lambda}_{\sigma}(\mathrm{S}-\mathrm{P})+Z_{\mathrm{VA}} \bar{\lambda}_{\mathrm{VA}}\left[2(\mathrm{~V}-\mathrm{A})^{\mathrm{adj}}+\left(1 / \mathrm{N}_{\mathrm{c}}\right)(\mathrm{V}-\mathrm{A})\right]\right] .
\end{aligned}
$$

Here $A_{\mu}=A^{z} T^{z}, F_{\mu \nu}=F_{\mu \nu}^{z} T^{z}$ denotes the nonabelian gauge potential and field strength, and $B_{\mu}, B_{\mu \nu}$ the abelian ones. The gauge-field kinetic terms are accompanied by wavefunction renormalizations $Z_{\mathrm{F}}$ and $Z_{\mathrm{B}}$, the fermionic one by $Z_{\psi}$. Similarly, $Z_{1}, Z_{1}^{\mathrm{B}}, Z_{+}, Z_{-}$, $Z_{\mathrm{f}}$ and $Z_{\mathrm{VA}}$ are the vertex renormalizations, whereas $\bar{e}, \bar{g}, \bar{\lambda}$ denote the bare couplings. The renormalized (dimensionless) couplings are defined as

$$
g=\frac{\bar{g} Z_{1}}{Z_{\mathrm{B}}^{1 / 2} Z_{\psi}}, \quad e=\frac{\bar{e} Z_{1}^{\mathrm{B}}}{Z_{\mathrm{F}}^{1 / 2} Z_{\psi}}, \quad \hat{\lambda}=\frac{Z_{\lambda} k^{2} \bar{\lambda}}{Z_{\psi}^{2}} .
$$

We work in the Landau gauge, $\alpha=\alpha_{B}=0$, which is known to be a fixed point of

\footnotetext{
${ }^{2}$ We note that only the four-fermion interactions are manifestly invariant under local gauge transformations for all possible choices of the couplings. Gauge invariance of the remaining terms is governed by (modified) Ward-Takahashi identities as discussed in Sect. [4
} 
the renormalization group [12] and has the additional advantage that the fermionic wave function is not renormalized in our truncation, such that we can choose $Z_{\psi}=1$.

The four-fermion interactions can be classified according to their color and flavor structure. Color and flavor singlets are

$$
\begin{aligned}
(\mathrm{V}-\mathrm{A}) & =\left(\bar{\psi} \gamma_{\mu} \psi\right)^{2}+\left(\bar{\psi} \gamma_{\mu} \gamma_{5} \psi\right)^{2} \\
(\mathrm{~V}+\mathrm{A}) & =\left(\bar{\psi} \gamma_{\mu} \psi\right)^{2}-\left(\bar{\psi} \gamma_{\mu} \gamma_{5} \psi\right)^{2}
\end{aligned}
$$

where color $(i, j, \ldots)$ and flavor $(a, b, \ldots)$ indices are contracted pairwise, e.g., $(\bar{\psi} \psi) \equiv$ $\left(\bar{\psi}_{i}^{a} \psi_{i}^{a}\right)$. The operators of non-trivial color or flavor structure are denoted by

$$
\begin{aligned}
(\mathrm{S}-\mathrm{P}) & =\left(\bar{\psi}^{a} \psi^{b}\right)^{2}-\left(\bar{\psi}^{a} \gamma_{5} \psi^{b}\right)^{2} \equiv\left(\bar{\psi}_{i}^{a} \psi_{i}^{b}\right)^{2}-\left(\bar{\psi}_{i}^{a} \gamma_{5} \psi_{i}^{b}\right)^{2}, \\
(\mathrm{~V}-\mathrm{A})^{\mathrm{adj}} & =\left(\bar{\psi} \gamma_{\mu} T^{z} \psi\right)^{2}+\left(\bar{\psi} \gamma_{\mu} \gamma_{5} T^{z} \psi\right)^{2},
\end{aligned}
$$

where we define $\left(\bar{\psi}^{a} \psi^{b}\right)^{2} \equiv \bar{\psi}^{a} \psi^{b} \bar{\psi}^{b} \psi^{a}$, etc., and $\left(T^{z}\right)_{i j}$ denotes the generators of the gauge group in the fundamental representation. Owing to Fierz identities, the last four-fermion structure in Eq. (3) can also be written as

$$
\left[2(\mathrm{~V}-\mathrm{A})^{\mathrm{adj}}+\left(1 / \mathrm{N}_{\mathrm{c}}\right)(\mathrm{V}-\mathrm{A})\right]=\left(\bar{\psi}_{i} \gamma_{\mu} \psi_{j}\right)^{2}+\left(\bar{\psi}_{i} \gamma_{\mu} \gamma_{5} \psi_{j}\right)^{2} \equiv\left(\bar{\psi}^{a} \gamma_{\mu} \psi^{b}\right)^{2}+\left(\bar{\psi}^{a} \gamma_{\mu} \gamma_{5} \psi^{b}\right)^{2}
$$

The set of four different fermionic self-interactions occurring in Eq. (3) forms a complete basis. Any other point-like four-fermion interaction invariant under $S U\left(N_{c}\right) \times U(1)$ gauge symmetry and $\mathrm{SU}\left(\mathrm{N}_{\mathrm{f}}\right)_{\mathrm{L}} \times \mathrm{SU}\left(\mathrm{N}_{\mathrm{f}}\right)_{\mathrm{R}}$ flavor symmetry can be decomposed into these base elements by means of Fierz transformations.

Evaluating the RG flow equation in the limit of point-like interactions and projecting the result onto our truncation (3), we obtain the following $\beta$ functions for the dimensionless couplings $\hat{\lambda}$ :

$$
\begin{aligned}
\partial_{t} \hat{\lambda}_{-}=\beta_{-}= & 2 \hat{\lambda}_{-}-4 v_{4} l_{1,1}^{(\mathrm{FB}), 4}\left[\left(\frac{3}{\mathrm{~N}_{\mathrm{c}}} g^{2}-3 e^{2}\right) \hat{\lambda}_{-}-3 g^{2} \hat{\lambda}_{\mathrm{VA}}\right] \\
& -\frac{1}{8} v_{4} l_{1,2}^{(\mathrm{FB}), 4}\left[\frac{12+9 \mathrm{~N}_{\mathrm{c}}^{2}}{\mathrm{~N}_{\mathrm{c}}^{2}} g^{4}+48 e^{4}-\frac{48}{\mathrm{~N}_{\mathrm{c}}} e^{2} g^{2}\right] \\
& -8 v_{4} l_{1}^{\mathrm{F}), 4}\left\{-\mathrm{N}_{\mathrm{f}} \mathrm{N}_{\mathrm{c}}\left(\hat{\lambda}_{-}^{2}+\hat{\lambda}_{+}^{2}\right)+\hat{\lambda}_{-}^{2}-2\left(\mathrm{~N}_{\mathrm{c}}+\mathrm{N}_{\mathrm{f}}\right) \hat{\lambda}_{-} \hat{\lambda}_{\mathrm{VA}}+\mathrm{N}_{\mathrm{f}} \hat{\lambda}_{+} \hat{\lambda}_{\sigma}+2 \hat{\lambda}_{\mathrm{VA}}^{2}\right\}, \\
\partial_{t} \hat{\lambda}_{+}=\beta_{+}= & 2 \hat{\lambda}_{+}-4 v_{4} l_{1,1}^{\mathrm{FB}), 4}\left[\left(-\frac{3}{\mathrm{~N}_{\mathrm{c}}} g^{2}+3 e^{2}\right) \hat{\lambda}_{+}\right] \\
& -\frac{1}{8} v_{4} l_{1,2}^{(\mathrm{FB}), 4}\left[-\frac{12+3 \mathrm{~N}_{\mathrm{c}}^{2}}{\mathrm{~N}_{\mathrm{c}}^{2}} g^{4}-48 e^{4}+\frac{48}{\mathrm{~N}_{\mathrm{c}}} e^{2} g^{2}\right] \\
& -8 v_{4} l_{1}^{(\mathrm{F}), 4}\left\{-3 \hat{\lambda}_{+}^{2}-2 \mathrm{~N}_{\mathrm{c}} \mathrm{N}_{\mathrm{f}} \hat{\lambda}_{-} \hat{\lambda}_{+}-2 \hat{\lambda}_{+}\left(\hat{\lambda}_{-}+\left(\mathrm{N}_{\mathrm{c}}+\mathrm{N}_{\mathrm{f}}\right) \hat{\lambda}_{\mathrm{VA}}\right)\right. \\
& \left.+\mathrm{N}_{\mathrm{f}} \hat{\lambda}_{-} \hat{\lambda}_{\sigma}+\hat{\lambda}_{\mathrm{VA}} \hat{\lambda}_{\sigma}+\frac{1}{4} \hat{\lambda}_{\sigma}^{2}\right\}
\end{aligned}
$$




$$
\begin{aligned}
\partial_{t} \hat{\lambda}_{\sigma}=\beta_{\sigma}= & 2 \hat{\lambda}_{\sigma}-4 v_{4} l_{1,1}^{(\mathrm{FB}), 4}\left[\left(6 C_{2}\left(\mathrm{~N}_{\mathrm{c}}\right) g^{2}+3 e^{2}\right) \hat{\lambda}_{\sigma}-6 g^{2} \hat{\lambda}_{+}\right] \\
& -\frac{1}{4} v_{4} l_{1,2}^{(\mathrm{FB}), 4}\left[-\frac{24-9 \mathrm{~N}_{\mathrm{c}}^{2}}{\mathrm{~N}_{\mathrm{c}}} g^{4}+48 e^{2} g^{2}\right] \\
& -8 v_{4} l_{1}^{(\mathrm{F}), 4}\left\{2 \mathrm{~N}_{\mathrm{c}} \hat{\lambda}_{\sigma}^{2}-2 \hat{\lambda}_{-} \hat{\lambda}_{\sigma}-2 \mathrm{~N}_{\mathrm{f}} \hat{\lambda}_{\sigma} \hat{\lambda}_{\mathrm{VA}}-6 \hat{\lambda}_{+} \hat{\lambda}_{\sigma}\right\} \\
\partial_{t} \hat{\lambda}_{\mathrm{VA}}=\beta_{\mathrm{VA}}= & 2 \hat{\lambda}_{\mathrm{VA}}-4 v_{4} l_{1,1}^{(\mathrm{FB}), 4}\left[\left(\frac{3}{\mathrm{~N}_{\mathrm{c}}} g^{2}-3 e^{2}\right) \hat{\lambda}_{\mathrm{VA}}-3 g^{2} \hat{\lambda}_{-}\right] \\
& -\frac{1}{8} v_{4} l_{1,2}^{(\mathrm{FB}), 4}\left[-\frac{24-3 \mathrm{~N}_{\mathrm{c}}^{2}}{\mathrm{~N}_{\mathrm{c}}} g^{4}+48 e^{2} g^{2}\right] \\
& -8 v_{4} l_{1}^{(\mathrm{F}), 4}\left\{-\left(\mathrm{N}_{\mathrm{c}}+\mathrm{N}_{\mathrm{f}}\right) \hat{\lambda}_{\mathrm{VA}}^{2}+4 \hat{\lambda}_{-} \hat{\lambda}_{\mathrm{VA}}-\frac{1}{4} \mathrm{~N}_{\mathrm{f}} \hat{\lambda}_{\sigma}{ }^{2}\right\}
\end{aligned}
$$

Here $C_{2}\left(\mathrm{~N}_{\mathrm{c}}\right)=\left(\mathrm{N}_{\mathrm{c}}^{2}-1\right) /\left(2 \mathrm{~N}_{\mathrm{c}}\right)$ is a Casimir operator of the gauge group, and $v_{4}=1 /\left(32 \pi^{2}\right)$. The $l$ quantities are positive constant numbers of $\mathcal{O}(1)$ that characterize the regulator dependence [13. For better readability, we have written all gauge-coupling-dependent terms in square brackets, whereas fermionic self-interactions are grouped inside curly brackets. For small gauge couplings, the running of $g$ and $e$ is governed by their standard perturbative $\beta$-functions - this will be discussed in more Detail in sect. 4

\section{$3 \quad$ Fixed points for purely fermionic models}

A fixed point corresponds to a simultaneous zero of all $\beta$-functions. Each fixed point defines a (nonperturbatively) renormalizable theory within our truncation. Each fixed point furthermore constitutes its own universality class. Let us first analyze the RG flow of the fermionic couplings $\hat{\lambda}_{i}$ given above in the simplified context of vanishing gauge couplings, $g^{2}, e^{2} \rightarrow 0$. Then, in the point-like approximation the $\beta$ functions are all of the same form:

$$
\partial_{t} \hat{\lambda}_{i}=\beta_{i}(\hat{\lambda})=(d-2) \lambda_{i}+\hat{\lambda}_{k} A_{i}^{k l} \hat{\lambda}_{l}
$$

where $A_{i}^{k l}$ are constant matrices which are symmetric in the upper indices, and we generalize the right-hand side formally to $d$ dimensional spacetime. For fixed $\hat{\lambda}_{j \neq i}$, the $\beta$ function for $\hat{\lambda}_{i}$ corresponds graphically to a parabola, such that the fixed-point equation $\partial_{t} \hat{\lambda}_{i}=$ $\beta_{i}\left(\hat{\lambda}_{j \neq i} ; \hat{\lambda}_{i}\right)=0$ has exactly two (possibly complex or degenerate) solutions for $\hat{\lambda}_{i}$. Since our truncation Eq. (3) has 4 fermionic couplings, we expect up to $2^{4}=16$ different fixed points. A computer-algebraical inspection of Eq. (111) indeed reveals these 16 fixed points, all of which are real and therefore physically acceptable ${ }^{3}$; we do not find degeneracies.

Consequently, in this framework each fixed point serves as a possibility to define a fundamental renormalizable quantum system in which the continuum limit can be taken. The triviality problem is thus absent; however, the hierarchy problem remains in these

\footnotetext{
${ }^{3}$ This is a particularity of the $\mathrm{SU}\left(\mathrm{N}_{\mathrm{f}}\right)_{\mathrm{L}} \times \mathrm{SU}\left(\mathrm{N}_{\mathrm{f}}\right)_{\mathrm{R}}$ flavor symmetry. For instance, a less restrictive $\mathrm{SU}\left(\mathrm{N}_{\mathrm{f}}\right)_{\mathrm{V}}$ flavor symmetry does allow for 6 different four-fermion couplings, implying $2^{6}=64$ fixed points, only 44 of which are real and physically acceptable.
} 
purely fermionic systems in the point-like limit. This can be shown by studying the stability matrix $B_{i}{ }^{j}$, defined by the derivatives of the $\beta$ functions at the fixed point

$$
B_{i}^{j}=\left.\frac{\partial \beta_{i}}{\partial \hat{\lambda}_{j}}\right|_{\hat{\lambda}_{*}}=(d-2) \delta_{i}^{j}+2 \hat{\lambda}_{* k} A_{i}^{k j} .
$$

The eigenvalues of the stability matrix and the associated eigenvectors $v$ govern the evolution of small deviations from the fixed point according to $B v=-\Theta v$. (We denote by $\Theta$ the negative of the eigenvalues). In turn, this determines the running of the couplings in the fixed-point regime, $\left(\hat{\lambda}-\hat{\lambda}_{*}\right) \sim\left(\Lambda_{\mathrm{UV}} / k\right)^{\Theta}$. Therefore, large positive $\Theta$ implies a rapid growth of the couplings towards the infrared and corresponds to a strongly relevant RG direction, indicating IR instability such as a scalar mass term.

In the present problem, a large positive eigenvalue $\Theta=(d-2)$ of the stability matrix exists for each fixed point $\hat{\lambda}_{*}$. This can been seen from the following argument: let $\hat{\lambda}_{*}$ be a solution of the fixed point equation,

$$
\partial_{t} \hat{\lambda}_{* i}=(d-2) \hat{\lambda}_{* i}+\hat{\lambda}_{* k} A_{i}^{k l} \hat{\lambda}_{* l}=0 \quad, \forall i
$$

Acting with $B_{i}{ }^{j}$ on $\hat{\lambda}_{* j} \neq 0$ we have

$$
\begin{aligned}
B_{i}{ }^{j} \hat{\lambda}_{* j} & =(d-2) \hat{\lambda}_{* i}+2 \hat{\lambda}_{* j} A_{i}^{j k} \hat{\lambda}_{* k} \\
& =-(d-2) \hat{\lambda}_{* i}+2\left((d-2) \hat{\lambda}_{* i}+\hat{\lambda}_{* j} A_{i}^{j k} \hat{\lambda}_{* k}\right) \\
& =-(d-2) \hat{\lambda}_{* i},
\end{aligned}
$$

where we have used the fixed-point equation in the last step. This shows that $\hat{\lambda}_{*}$ itself is an eigenvector of the stability matrix with the eigenvalue $-(d-2)$, hence $\Theta=(d-2)$. For $d=4$ we have therefore at least one "quadratically renormalizing" relevant direction in the fixed-point regime, which is the same RG behavior as for a system with a fundamental Higgs scalar. A very precise choice for the initial conditions at the high scale (GUT scale) is required in order to separate the high scale from the symmetry-breaking scale (Fermi scale).

The second lesson to be learned from Eq. (14) is that there cannot be a purely infrared attractive fixed point besides $\hat{\lambda}=0$ in this truncation. In other words, all remaining 15 fixed points can be used for defining an interacting continuum limit, which requires at least one IR repulsive (or marginal) direction.

The fixed points can be classified further according to their number of relevant and irrelevant directions, constituting the number of physical parameters. In our case, the number of fixed points with $j$ relevant directions turns out to be equal to the binomial coefficient $\left(\begin{array}{l}4 \\ j\end{array}\right)$. There is one IR stable fixed point (only irrelevant directions) which is the Gaußian fixed point, and also exactly one fixed point with only relevant directions which is IR repulsive in all directions in this truncation. All other fixed points have relevant and irrelevant directions. This is illustrated in the left panel of Fig. 1 using the subsystem 

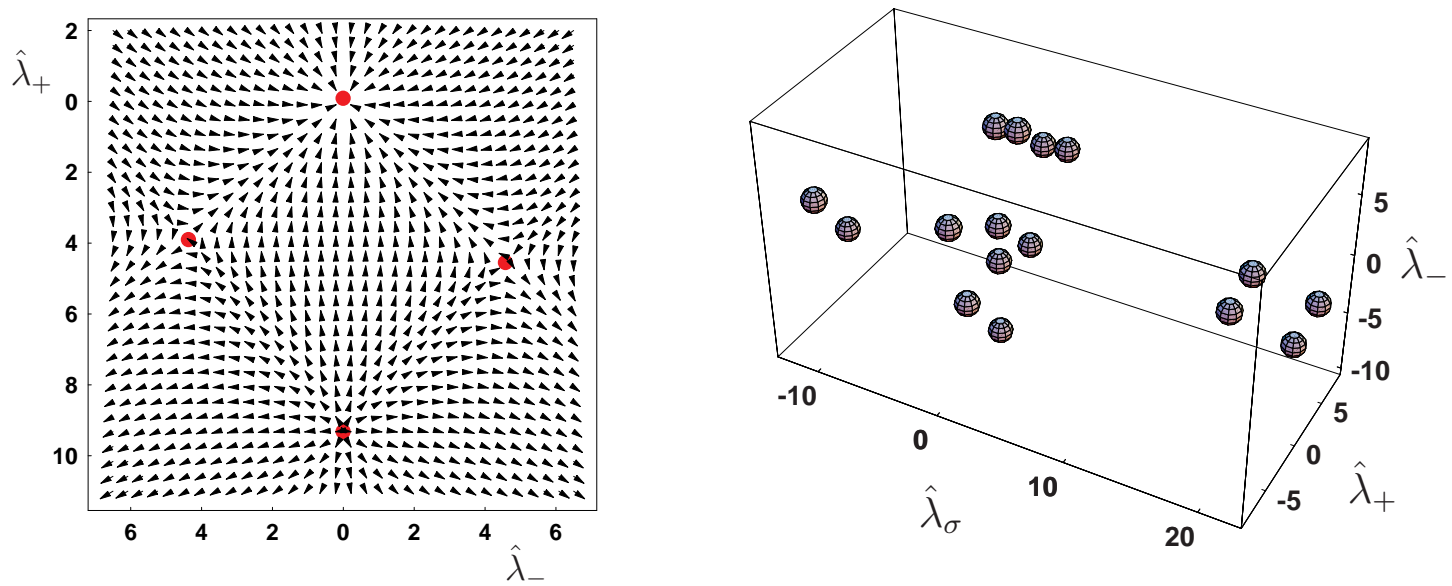

Figure 1: Left panel: RG flow in the $\left(\hat{\lambda}_{+}, \hat{\lambda}_{-}\right)$subsystem. The filled circles (red) mark the fixed points, and the arrows show the direction of the flow toward the infrared. It is easy to see that only the Gaußian fixed point, $\hat{\lambda}_{+}=\hat{\lambda}_{-}=0$, is IR stable. All others have at least one relevant, IR repulsive direction. Right Panel: all 16 fixed points of the full system projected onto the $\left(\hat{\lambda}_{+}, \hat{\lambda}_{-}, \hat{\lambda}_{\sigma}\right)$ subspace.

$\hat{\lambda}_{+}, \hat{\lambda}_{-}$as a simple example (further parameters: $d=4, \mathrm{~N}_{\mathrm{c}}=3, \mathrm{~N}_{\mathrm{f}}=6$, and linear cutoff functions [14] $\left.\left(l_{1}^{(\mathrm{F}), 4}=1 / 2, l_{1,1}^{(\mathrm{FB}), 4}=1, l_{1,2}^{(\mathrm{FB}), 4}=3 / 2\right)\right)$. The right panel of Fig. 11 displays all 16 fixed points projected onto the $\left(\hat{\lambda}_{+}, \hat{\lambda}_{-}, \hat{\lambda}_{\sigma}\right)$ subspace.

\section{Gauge interactions}

Let us now include the gauge interactions in our considerations. For this, we need the running of the gauge couplings which we derive from the fermion-gauge-field vertex $\Gamma_{\mu}$. For instance, in the abelian case, the general form of this vertex is

$$
\Gamma_{\bar{\psi} \psi B}=\bar{e} \int_{q_{1}, q_{2}} \bar{\psi}\left(q_{2}\right) \Gamma_{\mu}\left(q_{2}, q_{1}\right) B_{\mu}\left(q_{2}-q_{1}\right) \psi\left(q_{1}\right)
$$

from which we define the renormalized coupling $e$ in the Thompson limit,

$$
\lim _{p \rightarrow 0} \Gamma_{\mu}(q, q+p)=Z_{\psi} \frac{e}{\bar{e}} Z_{\mathrm{F}}^{1 / 2} \gamma_{\mu}=Z_{1}^{\mathrm{B}} \gamma_{\mu}
$$

and similarly for the nonabelian coupling $g$. (Here we included the fermion wave-function renormalization $Z_{\psi}$ for full generality, but, as we already mentioned, with Landau gauge we have $Z_{\psi}=1$ in our truncation.) Within our truncation, the flow equation for the vertex 


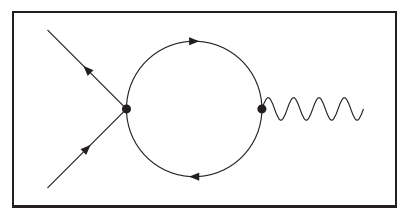

Figure 2: Correction to the gauge-boson-fermion vertex (fermions solid with arrow, gauge boson wiggled) in the presence of a four-fermion interaction.

results in the $\beta$ functions

$$
\begin{aligned}
& \partial_{t} g^{2}=\eta_{\mathrm{F}} g^{2}-8 v_{4} l_{1}^{(\mathrm{F}), 4}[ {\left[\hat{\lambda}_{\sigma}-2 \hat{\lambda}_{-}+\mathrm{N}_{\mathrm{f}} \hat{\lambda}_{\sigma}-2 \mathrm{~N}_{\mathrm{f}} \hat{\lambda}_{\mathrm{VA}}\right] g^{2} } \\
& \partial_{t} e^{2}=\eta_{\mathrm{B}} e^{2}-8 v_{4} l_{1}^{(\mathrm{F}), 4}\left[\hat{\lambda}_{\sigma}-2 \hat{\lambda}_{-}-2 \mathrm{~N}_{\mathrm{f}} \mathrm{N}_{\mathrm{c}}\left(\hat{\lambda}_{+}+\hat{\lambda}_{-}\right)\right. \\
&\left.+\left(\mathrm{N}_{\mathrm{c}} \hat{\lambda}_{\sigma}^{\mathrm{c}}+\mathrm{N}_{\mathrm{f}} \hat{\lambda}_{\sigma}\right)-2\left(\mathrm{~N}_{\mathrm{c}}+\mathrm{N}_{\mathrm{f}}\right) \hat{\lambda}_{\mathrm{VA}}\right] e^{2}
\end{aligned}
$$

where the standard one-loop coefficients are contained in the anomalous dimensions of the gauge field,

$$
\begin{array}{ll}
\eta_{\mathrm{F}}=-\frac{1}{Z_{\mathrm{F}}} \partial_{t} Z_{\mathrm{F}}=-4 v_{4} b_{0}^{g^{2}} g^{2}, & b_{0}^{g^{2}}=\frac{11}{3} \mathrm{~N}_{\mathrm{c}}-\frac{2}{3} \mathrm{~N}_{\mathrm{f}} \\
\eta_{\mathrm{B}}=-\frac{1}{Z_{\mathrm{B}}} \partial_{t} Z_{\mathrm{B}}=-4 v_{4} b_{0}^{e^{2}} e^{2}, & b_{0}^{e^{2}}=-\frac{4}{3} \mathrm{~N}_{\mathrm{f}} \mathrm{N}_{\mathrm{c}}
\end{array}
$$

The additional $\hat{\lambda}$-dependent terms in Eqs. (17), (18) arise from diagrams of the form shown in Fig. 2. At first sight, it seems that these additional terms offer a new and rich structure for the possible UV behavior of the system. For instance, for a given non-Gaußian $\hat{\lambda}$ fixed point $\hat{\lambda}_{*}$, these terms are dominant in the small gauge coupling limit. If the factor in square brackets in these terms is positive for a given $\hat{\lambda}_{*}$, the corresponding gauge coupling seems to be asymptotically free. This would offer a solution to the triviality problem of the $\mathrm{U}(1)$ sector. Moreover, the interplay of both terms on the right-hand side of these $\beta$ functions can produce non-Gaußian fixed points in the gauge couplings. This would not only be a possible solution of triviality, but could also lead to a sizeable reduction of the critical exponents and the hierarchy problem by circumventing the argument of Eq. (14).

However, these hopes can unfortunately not be confirmed, as quantum field theory tells us in the following interesting way. Equations (17) and (18) are not the only source of information about the vertex that we can obtain from the flow-equation formalism. The requirement of gauge invariance is encoded in a constraint for the effective action: the Ward-Takahashi identity (WTI). Since the regulator of the flow equation formalism is not manifestly gauge invariant, it also contributes to the constraint, leading to a modified Ward-Takahashi identity (mWTI) [15].

For simplicity, let us analyze the mWTI arising from abelian gauge symmetry. Employing the generator $\mathcal{G}$ of an infinitesimal gauge transformation (in momentum space), 


$$
\mathcal{G}(p)=i p_{\mu} \frac{\delta}{\delta B_{\mu}(-p)}-i \bar{e}\left[\int_{q} \psi(q) \frac{\delta}{\delta \psi(q-p)}-\int_{q} \bar{\psi}(q) \frac{\delta}{\delta \bar{\psi}(q+p)}\right],
$$

the mWTI can be written as

$\mathcal{G}(p) \Gamma_{k}-\frac{i}{\alpha_{B}} p^{2} p_{\mu} B_{\mu}(p)=-i \bar{e} \operatorname{tr} \int_{q}\left[R_{k}^{\psi}(q+p) G_{\psi \bar{\psi}}(q+p, q)-R_{k}^{\psi T}(q+p) G_{\bar{\psi}^{T} \psi^{T}}(q+p, q)\right]$,

where, e.g., $G_{\psi \bar{\psi}}=\left(\Gamma^{(2)}+R_{k}\right)_{\psi \bar{\psi}}^{-1}$ denotes the $(\psi \bar{\psi})$ component of the propagator, and the $T$ symbol indicates transposition in Dirac space. For vanishing regulator $R_{k} \rightarrow 0$, the righthand side of the mWTI is zero and we rediscover the standard WTI. However, since we are dealing with a perturbatively nonrenormalizable theory, the presence of the regulator is essential in order to define the quantum theory, i.e., the Schwinger functional. Therefore, the right-hand side of the mWTI should not only be viewed as a technical complication, but as important information about the structure of the theory.

Owing to its similar structure, the mWTI can be evaluated with the same technology as the flow equation; the right-hand side, e.g., is again of one-loop form with an exact propagator in the loop. Information about the running gauge coupling can be found by projecting the mWTI onto the operator $\sim \bar{\psi} \psi$, yielding

$$
e=\bar{e} Z_{\mathrm{B}}^{-1 / 2}\left(1-2 v_{4} l_{1}^{(\mathrm{F}), 4} \sum c_{i}^{e} \hat{\lambda}_{i}\right)
$$

i.e.,

$$
\frac{Z_{1}^{\mathrm{B}}}{Z_{\psi}}=\left(1-2 v_{4} l_{1}^{(\mathrm{F}), 4} \sum c_{i}^{e} \hat{\lambda}_{i}\right)
$$

where we abbreviated the combination

$$
\sum c_{i}^{e} \hat{\lambda}_{i}:=\hat{\lambda}_{\sigma}-2 \hat{\lambda}_{-}-2 \mathrm{~N}_{\mathrm{f}} \mathrm{N}_{\mathrm{c}}\left(\hat{\lambda}_{+}+\hat{\lambda}_{-}\right)+\left(\mathrm{N}_{\mathrm{c}} \hat{\lambda}_{\sigma}^{\mathrm{c}}+\mathrm{N}_{\mathrm{f}} \hat{\lambda}_{\sigma}\right)-2\left(\mathrm{~N}_{\mathrm{c}}+\mathrm{N}_{\mathrm{f}}\right) \hat{\lambda}_{\mathrm{VA}}
$$

as it also occurs in Eq. (18). In ordinary QED, the term $\sim \hat{\lambda}$ is not present and we end up with the standard result that the running of the coupling corresponds to the running of the gauge-field wave-function renormalization ${ }^{4}, e=\bar{e} Z_{\mathrm{B}}^{-1 / 2}$. The presence of the fermionic interactions replaces this simple relation by Eq. (22).

By differentiation, we obtain the $\beta$ function

$$
\partial_{t} e^{2}=\eta_{\mathrm{B}} e^{2}-4 v_{4} l_{1}^{(\mathrm{F}), 4} \frac{e^{2}}{1-2 v_{4} l_{1}^{(\mathrm{F}), 4} \sum c_{i}^{e} \hat{\lambda}_{i}} \partial_{t} \sum c_{i}^{e} \hat{\lambda}_{i} .
$$

Here $\partial_{t} \sum c_{i}^{e} \hat{\lambda}_{i}$ means that we have to insert the complete $\beta$ functions for the $\hat{\lambda}_{i}$ into Eq. (25). Since $\partial_{t} \sum c_{i}^{e} \hat{\lambda}_{i}=2 \sum c_{i}^{e} \hat{\lambda}_{i}+\ldots$ in a small-coupling expansion, we rediscover

\footnotetext{
${ }^{4}$ With Eq. (23), this corresponds to the standard result $Z_{1}^{\mathrm{B}}=Z_{\psi}$. We remark that from Eq. (23) alone only the ratio of $Z_{\psi}$ and $Z^{\mathrm{B}}$ is accessible (and physically relevant). Still, in approximations it can make a difference if $Z_{\psi}$ or $Z_{1}^{\mathrm{B}}$ is running. Consistent with our choice of the Landau gauge, we set $Z_{\psi}=1$.
} 
the result of the flow equation (18) in this limit. In other words, flow equation and mWTI agree within the order of our truncation, as they should. The mWTI, however, contains considerably more information, since the non-monomial appearance of the $\hat{\lambda}$ 's suggests that the mWTI represents a resummation of a larger class of diagrams.

This has important consequences for the RG behavior of the gauge coupling, if compared to the possibilities that are offered by the simpler form of Eq. (18). At the non-Gaußian $\hat{\lambda}$ fixed points, the $\hat{\lambda}$ flow vanishes, such that $\partial_{t} \sum c_{i}^{e} \hat{\lambda}_{i} \rightarrow 0$. This implies that the running of the gauge coupling in the vicinity of its Gaußian fixed point is determined by the standard one-loop term $\eta_{\mathrm{B}} e^{2}$ alone. The fermionic self-interactions contribute only to higher order. ${ }^{5}$ The abelian gauge coupling can therefore not be rendered asymptotically free by the influence of the four-fermion interactions. By a similar argument, the additional term in Eq. (25) does not facilitate the existence of non-Gaußian fixed points, $e_{*} \neq 0$, in the abelian gauge coupling within this truncation. Again, if the $\hat{\lambda}^{\prime}$ s approach a fixed point, $\partial_{t} \sum c_{i}^{e} \hat{\lambda}_{i} \rightarrow 0$ and we are left with the standard running only, for which no non-Gaußian fixed point is known.

To summarize, the four-fermion contributions to the running of the abelian gauge coupling are not capable of solving the triviality problem in our truncation.

Let us finally comment on the RG flow of the running $\mathrm{SU}\left(\mathrm{N}_{\mathrm{c}}\right)$ coupling $g^{2}$. Although the mWTI for the nonabelian sector has a more complex structure, the result for the fourfermion contribution to the running gauge coupling has the same form as in Eq. (25). Near the Gaußian fixed point, the standard one-loop running holds and the non-abelian gauge sector remains asymptotically free. Actually, this finding is in line with another argument: we could equally well define the running of the nonabelian gauge coupling by the threegauge-boson vertex. At one-loop order, there is no contribution to the renormalization of this vertex from the four-fermion couplings $\hat{\lambda}$. As a consequence, the usual one-loop $\beta$ function governs the flow of this three-gluon vertex.

\section{$5 \quad$ Spontaneous symmetry breaking}

In order to illustrate the flow of the system from a non-Gaußian fixed point towards the regime of spontaneous symmetry breaking, we select a fixed point with only one relevant direction (for instance, the fixed point on the right-hand side or left-hand side in the left panel of Fig. 10. At the high scale $\Lambda_{\mathrm{UV}}$, we specify initial conditions such that all couplings in our truncation are roughly in the vicinity of their fixed point values; no fine-tuning is needed for this step. If we now compute the RG flow towards the infrared, the system either relaxes towards the Gaußian fixed point with $\hat{\lambda} \rightarrow 0$ as long as the gauge couplings are weak; or the system rapidly approaches the regime of spontaneous symmetry breaking (SSB) which is signaled by diverging four-fermion couplings. In the former case, the system is in the universality class of $\mathrm{SU}\left(\mathrm{N}_{\mathrm{c}}\right) \times \mathrm{U}(1)$ gauge theory with $\mathrm{N}_{\mathrm{f}}$ chiral fermions: in the

\footnotetext{
${ }^{5}$ A careful analysis reveals that the additional term in Eq. (25) is of the same order as the two-loop running of the gauge coupling for small perturbations around the fixed point.
} 

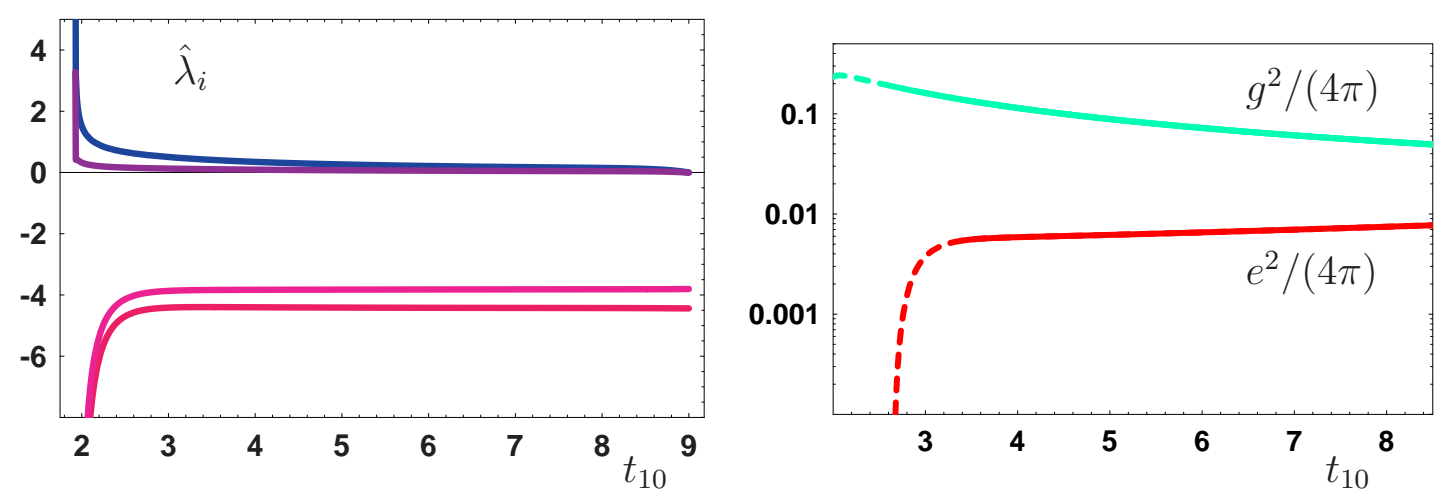

Figure 3: Running couplings as a function of the renormalization scale $k=10^{t_{10}} \mathrm{GeV}$. Left panel: flow of the four-fermion couplings $\hat{\lambda}_{\sigma}, \hat{\lambda}_{\mathrm{VA}}, \hat{\lambda}_{-}, \hat{\lambda}_{+}$(from top to bottom); the divergence of the couplings near the Fermi scale $10^{t_{10}} \sim 10^{2}$ signals the approach to SSB. Right panel: flow of the gauge couplings; while the $\hat{\lambda}_{i}$ are close to their fixed-point values, the gauge couplings run according to standard perturbation theory. The rapid behavior near the Fermi scale is likely to be an artefact of the truncation (dashed lines).

latter case we are dealing with a universality class characterized by SSB. Finally, we finetune only one of the parameters of the initial conditions such that the system is very close to the phase boundary on the SSB side. This fine-tuning corresponds effectively to a determination of the scale $k_{\mathrm{SSB}}$ at which the system runs into the SSB regime.

In Fig. 3. we display a particular solution to the flow equation obtained in the aforementioned way for $\mathrm{N}_{\mathrm{c}}=3, \mathrm{~N}_{\mathrm{f}}=6$ and a linear regulator [14. We have adjusted the gauge couplings roughly to their standard model values and fixed the four-fermion couplings to the value of one of the four non-Gaußian fixed points with one relevant direction. Finally, we have fine-tuned $\hat{\lambda}_{\text {VA }}$ so that the SSB scale corresponds to the Fermi scale $\sim 10^{2} \mathrm{GeV}$. On the left panel, the running of the four-fermion couplings from $k=10^{9} \mathrm{GeV}$ down to the Fermi scale is depicted. Over a wide range of scales, these couplings remain close to their fixed-point values with a slight modulation induced by the logarithmic increase of the gauge couplings. Near the Fermi scale, the running induced by the relevant direction becomes fast and the four-fermion couplings diverge, which signals the onset of SSB. With the present truncation, however, we cannot enter the SSB regime where the dynamics is governed by composite bosonic fluctuations on top of bosonic condensates $\sim\langle\bar{\psi} \psi\rangle$. A suitable description can be given by means of partial bosonization under the RG flow 16. In the present case, this would relate, e.g., the coupling $\hat{\lambda}_{\sigma}$ to a Yukawa coupling $h_{\sigma}$ and a mass term $m_{\sigma}$ for the composite scalar, $\hat{\lambda}_{\sigma} \sim h_{\sigma}^{2} / m_{\sigma}^{2}$. In this sense, the increase of $\lambda_{\sigma}$ is associated with a decrease of the scalar mass term, which eventually drops below zero and thus gives rise to SSB.

Using partial bosonization, one can moreover study the nature of the condensate, whereas in the present purely fermionic description we cannot distinguish the behavior of the various fermionic interaction channels. Owing to the nonlinear interplay of the flow equations for the couplings, all diverge simultaneously in this truncation. Even the gauge couplings can be affected, as is the case in our example for the abelian gauge coupling (see 
right panel of Fig. 3). This is clearly an artefact of the present truncation, and we expect a well-controllable flow once the threshold behavior is accounted for by using the techniques of [16], 17].

\section{Conclusions}

In this letter, we have analyzed the RG behavior of a standard-model-like system with purely fermionic matter content. The Higgs sector is replaced by fermionic self-interactions which are responsible for spontaneous symmetry breaking. Whereas the low-energy side of our models is reminiscent, and in the spirit, of topquark-condensation scenarios, we here concentrate on the UV behavior of such systems, investigating their renormalizability and RG stability in the framework of RG flow equations.

Within our truncation of point-like four-fermion interactions, we have identified a large number of non-Gaußian fixed points in the fermionic interactions, each of which constitutes an independent universality class with the given gauge and flavor symmetries at hand. From the structure of the flow equations, we deduce that, for $n$ independent four-fermion interactions, there exist up to $2^{n}$ fixed points. Each fixed point can serve to define an interacting continuum limit. We find no sign of triviality in our truncation in this fermionic Higgs sector. Contrary to the standard scalar Higgs sector, the fermionic systems have the potential to be valid down to arbitrarily small distances.

Furthermore, we have analyzed the RG stability of the model towards the IR. As a result, all fixed points with non-vanishing four-fermion interaction exhibit one RG relevant direction with critical exponent 2, i.e., renormalizing quadratically, similar to a fundamental scalar. Therefore, our fermionic models suffer from the same hierarchy problem as the conventional Higgs sector.

These findings are not modified by the inclusion of weakly coupled gauge interactions which only induce small anomalous dimensions. In turn, the fermionic self-interactions do not modify the leading-order running of the gauge couplings, as we have shown with the help of modified Ward-Takahashi identities. This inhibits a solution of the triviality problem in the abelian gauge sector at the Gaußian fixed point.

In summary, we find that a fermionic Higgs sector has the potential to be a truly renormalizable theory, removing the triviality problem of a fundamental scalar Higgs. However, we have not been able to identify a consistent resolution of the hierarchy problem within our truncation. In a realistic scenario comprising the full standard-model phenomenology, the number of physical parameters in our model would be comparable to that of the standard model. The precise number will depend on the particular choice of the non-Gaußian $\lambda_{*}$ fixed point and its number of RG-relevant directions. Whether the number of physical parameters can even drop below that of the standard model then depends on the universality class associated with the chosen fixed point. A determination of the physically acceptable universality classes requires an analysis of their symmetry-breaking properties. This is beyond the scope of the present truncation in which all couplings diverge at the symmetry-breaking scale. These low-energy properties can, however, easily be derived from 
an analysis of the condensing bilinear fermion channels using partial bosonization under the flow as described in [16], 17].

At this point, let us comment on the stability of our results under a change of the truncation. We have checked that higher fermionic self-interactions do not modify our results in the point-like limit. They neither remove the $\lambda_{*}$ fixed points nor represent nonperturbatively renormalizable couplings themselves (the latter would increase the number of physical parameters). Concerning the gauge sector, we have studied a number of non-minimal fermion-gauge-field couplings. None of them turns out to influence the leading-order running of the gauge couplings in the weak-coupling regime; the argument proceeds similarly to the one given in Sect. 4 based on the mWTI.

The UV fixed points that we find for the fermionic couplings may be viewed as a generalization of those UV fixed points that are known from large- $\mathrm{N}_{\mathrm{f}}$ studies of simple four-fermion interactions in $d=2+1$ dimensions [18. In the latter case, four-fermion interactions can be renormalized order by order in a $1 / \mathrm{N}_{\mathrm{f}}$ expansion despite its seeming perturbative nonrenormalizability. Our truncation exhibits these fixed points in all dimensions $d>2$. However, we suspect that at least far beyond four dimensions the fixed points may be an artefact of the truncation, since here even the induced Yukawa couplings between fermions and composite bosonic fluctuations become RG irrelevant by power-counting arguments. In $d=4$, these Yukawa couplings are RG marginal by power-counting: hence $d=4$ appears to be the critical dimension. Large- $\mathrm{N}_{\mathrm{f}}$ arguments are indeed in favor of logarithmic triviality in $d=4$, a picture that receives some support from lattice simulations with staggered fermions for a simple NJL model [19]. However, the large- $\mathrm{N}_{\mathrm{f}}$ approximation neglects the anomalous dimension of the fermion which, even if tiny, can have a large effect by changing marginal-irrelevant into marginal-relevant operators. Since gauge interactions also contribute to the fermionic anomalous dimension, purely fermionic lattice studies cannot be conclusive for the models considered in the present work. Therefore, $d=4$ lattice investigations with four-fermion as well as gauge interactions would be desirable and may indeed be accessible with recently developed algorithms [20].

In order to pursue this question further within the flow equation framework, we have to go beyond the point-like limit. If the qualitative picture developed so far in this simple truncation turns out to be incomplete, we expect that strong modifications might arise from the full momentum structure of the interaction. It may well be that our non-Gaußian $\lambda_{*}$ fixed points are only a projection of a more general momentum-dependent interaction onto the point-like limit. If so, it is natural to speculate that a strongly momentum-dependent wave-function renormalization of the fermions could even induce a large fermionic anomalous dimension. Then it would be conceivable that the latter stabilizes the fermionic flow towards the infrared. This would pave the way for a possible solution of the hierarchy problem in models with purely fermionic matter content. Concerning the hierarchy problem, another speculative alternative based on the assumed existence of a non-Gaußian fixed point for the gauge couplings has been investigated in the appendix. Whether or not one of these scenarios can indeed be realized is subject to further nonperturbative studies for which RG flow equations offer an appropriate framework. 


\section{Acknowledgment}

The authors are grateful to C.S. Fischer and J.M. Pawlowski for useful discussions. H.G. and J.J. acknowledge financial support by the Deutsche Forschungsgemeinschaft under contract Gi 328/1-2.

\section{A Non-Gaußian gauge systems}

Up to this point, our analysis reveals that a construction of standard-model-like theories based on non-Gaußian $\hat{\lambda}$ fixed points and Gaußian gauge fixed points still suffers from a hierarchy problem in the four-fermion sector as well as triviality of the abelian gauge sector - only triviality in the Higgs-like sector would be avoided in this scenario. In the following, we would like to demonstrate that the existence of a non-Gaußian fixed point in the abelian gauge coupling has the potential to solve both problems simultaneously.

Although the $\beta$ function for the abelian coupling $e^{2}$ as derived from the mWTI does not furnish a non-Gaußian fixed point via the direct four-fermion contribution, such a fixed point might be induced by the strong-coupling behavior of the gauge interactions or a combination of strong gauge and four-fermion interactions. This would manifest itself in a second zero of the anomalous dimension $\eta_{\mathrm{B}}\left(e_{*}\right)=0$ at nonzero $e_{*} \neq 0$. The search for such a non-Gaußian fixed point has a long tradition in the literature. Lattice studies of noncompact abelian gauge systems using staggered fermions [21] have not found such a fixed point; on the contrary, numerical data is compatible with logarithmic triviality. However, lattice results for field theories with both gauge and four-fermion couplings are not yet available, although perhaps accessible with recently developed algorithms [20]. Indications for the existence of such a fixed point in a gauged NJL model have been collected using Dyson-Schwinger equations [22]. Of course, the UV behavior of system as complex as the ones considered in this work is a completely open problem.

Therefore, let us from now on assume that such a fixed point $e_{*}>0$ in the abelian gauge coupling exists, and study its consequences. Since the $\beta_{e^{2}}$ function for $e^{2}$ is positive for small coupling, this non-Gaußian fixed point is necessarily UV stable, which solves the triviality problem. In order to study the hierarchy problem, we have to compute the dependence of the critical exponents for the $\hat{\lambda}$ 's on $e_{*}$. For a first impression, we perform the same numerical analysis as in Sect. 3. but now with nonzero $e=e_{*}$. Thereby, we neglect any possible influence of the contributions of the unknown $\beta_{e^{2}}$ function on the off-diagonal elements of the stability matrix; this is justified if the $\hat{\lambda}$ couplings do not play a dominant role for $\beta_{e^{2}}$ near $e=e_{*}$.

In Fig. 4, we display the dependence of the critical exponents on the hypothetical value of the fixed point $e_{*}$. Each line in these plots represents the maximal (most unstable) eigenvalue for a given non-Gaußian fixed point. As can be seen from the left panel 4(a), there is one non-Gaußian fixed point whose maximal eigenvalue decreases with increasing $e_{*}$ and can become close to zero if $\alpha_{*} \lesssim \alpha_{\text {cr }} \simeq 1.3$. At $\alpha_{\text {cr }}$, this fixed point annihilates with the Gaußian fixed point and disappears from the physically acceptable set. 

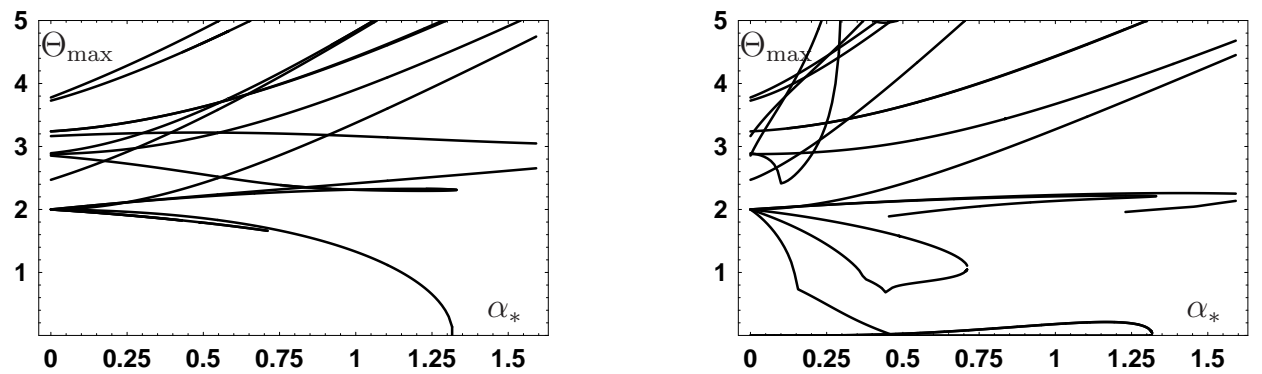

Figure 4: Maximal eigenvalue $\Theta_{\max }$ of the various fixed points of the four-fermion coupling depending on the assumed fixed-point value $\alpha_{e *}=\frac{e_{*}^{2}}{4 \pi}$ for the abelian gauge coupling (at $\left.\alpha_{g *}=\frac{g_{*}^{2}}{4 \pi}=0\right)$. The left panel shows the eigenvalue of the submatrix in the pure fourfermion sector while the right panel depicts the eigenvalue for the full matrix but with the unknown matrix elements in the pure gauge sector equal to zero.

In general, Fig. 4 does not represent the complete situation, since four-fermion couplings and gauge couplings mix nontrivially at the non-Gaußian fixed points. Technically speaking, we should not neglect the $\partial\left(\partial_{t} e^{2}\right) / \partial \lambda$ contributions to the stability matrix, nor $\partial\left(\partial_{t}\left(e^{2}, g^{2}\right)\right) / \partial\left(e^{2}, g^{2}\right)$. Whereas we can read off the former from the mWTI (25), nothing is known about the latter near the speculative fixed point $e_{*}$. Since we do not want to introduce a fine-tuning problem through the backdoor in this sector, it is natural to assume that these entries in the stability matrix are small. If this assumption is not valid, a large maximal eigenvalue will probably arise from this sector, and the present speculation is meaningless.

Therefore, we simply set the pure gauge entries to zero and study the evolution of the eigenvalues including the $\partial\left(\partial_{t} e^{2}\right) / \partial \lambda$ terms. The result is shown on the right panel of Fig. 4. Obviously, the mixing between the couplings exerts a strong quantitative influence on the eigenvalues. We find a whole range of possible $e_{*}$ fixed-point values for which the maximal eigenvalue of the stability matrix is small. The existence of such a non-Gaußian gauge fixed point therefore has the potential to stabilize the flow towards the IR significantly. The running towards the Fermi scale would then proceed with a small power or even almost logarithmically as for a system with marginal couplings only. In such a scenario, the hierarchy problem would be absent.

\section{References}

[1] C. Wetterich, Phys. Lett. B 140 (1984) 215.

[2] H. P. Nilles, Phys. Rept. 110, 1 (1984);

S. Weinberg, "The Quantum Theory Of Fields. Vol. 3: Supersymmetry," Cambridge, UK, (2000)

[3] E. Farhi and L. Susskind, Phys. Rept. 74, 277 (1981);

K. Lane, "Two lectures on technicolor," hep-ph/0202255 
[4] N. Arkani-Hamed, A. G. Cohen and H. Georgi, Phys. Lett. B 513 (2001) 232 hep-ph/0105239;

N. Arkani-Hamed, A. G. Cohen, T. Gregoire and J. G. Wacker, JHEP 0208 (2002) 020 hep-ph/0202089;

N. Arkani-Hamed, A. G. Cohen, E. Katz, A. E. Nelson, T. Gregoire and J. G. Wacker, JHEP 0208 (2002) 021 hep-ph/0206020;

I. Low, W. Skiba and D. Smith, Phys. Rev. D 66 (2002) 072001 hep-ph/0207243;

M. Schmaltz, Nucl. Phys. Proc. Suppl. 117 (2003) 40 hep-ph/0210415.

[5] Y. Nambu and G. Jona-Lasinio, Phys. Rev. 122, 345 (1961); ibid. 124, 246 (1961).

[6] V. A. Miransky, M. Tanabashi and K. Yamawaki, Phys. Lett. B 221, 177 (1989); Mod. Phys. Lett. A 4, 1043 (1989); W. A. Bardeen, C. T. Hill and M. Lindner, Phys. Rev. D 41, 1647 (1990);

for a review, see G. Cvetic, Rev. Mod. Phys. 71, 513 (1999) hep-ph/9702381.

[7] S. Weinberg, in C76-07-23.1 HUTP-76/160, Erice Subnucl. Phys., 1, (1976).

[8] C. Wetterich, Phys. Lett. B 104 (1981) 269, S. Bornholdt and C. Wetterich, Phys. Lett. B 282, 399 (1992).

[9] J. M. Kosterlitz and D. J. Thouless, J. Phys. CC 6 (1973) 1181.

[10] G. Von Gersdorff and C. Wetterich, Phys. Rev. B 64, 054513 (2001) hep-th/0008114.

[11] C. Wetterich, Phys. Lett. B 301, 90 (1993); Nucl. Phys. B 352, 529 (1991); Z. Phys. C 48, 693 (1990).

[12] U. Ellwanger, M. Hirsch and A. Weber, Z. Phys. C 69, 687 (1996) hep-th/9506019;

D. F. Litim and J. M. Pawlowski, Phys. Lett. B 435, 181 (1998) hep-th/9802064.

[13] J. Berges, N. Tetradis and C. Wetterich, Phys. Rept. 363, 223 (2002) hep-ph/0005122.

[14] D. F. Litim, Phys. Lett. B 486, 92 (2000) hep-th/0005245; Phys. Rev. D 64, 105007 (2001) hep-th/0103195.

[15] U. Ellwanger, Phys. Lett. B 335, 364 (1994) hep-th/9402077;

M. Reuter and C. Wetterich, Nucl. Phys. B 417, 181 (1994);

F. Freire, D. F. Litim and J. M. Pawlowski, Phys. Lett. B 495, 256 (2000) hep-th/0009110.

[16] H. Gies and C. Wetterich, Phys. Rev. D 65, 065001 (2002) hep-th/0107221; Acta Phys. Slov. 52, 215 (2002) hep-ph/0205226; hep-th/0209183.

[17] J. Jäckel and C. Wetterich, Phys. Rev. D 68, 025020 (2003) hep-ph/0207094;

J. Jäckel, hep-ph/0309090.

[18] B. Rosenstein, B. J. Warr and S. H. Park, Phys. Rev. Lett. 62, 1433 (1989);

K. Gawedzki and A. Kupiainen, Phys. Rev. Lett. 55, 363 (1985);

C. de Calan, P. A. Faria da Veiga, J. Magnen and R. Seneor, Phys. Rev. Lett. 66, 3233 (1991).

[19] S. Kim, A. Kocic and J. B. Kogut, Nucl. Phys. B 429, 407 (1994) hep-lat/9402016.

[20] S. Kim, J. B. Kogut and M. P. Lombardo, Phys. Rev. D 65, 054015 (2002) hep-lat/0112009.

[21] M. Gockeler, R. Horsley, V. Linke, P. Rakow, G. Schierholz and H. Stuben, Phys. Rev. Lett. 80, 4119 (1998) hep-th/9712244.

[22] M. Reenders, Phys. Rev. D 62, 025001 (2000) hep-th/9908158. 